\title{
Las violencias contra las mujeres en los textos jurídicos de América Latina y el Caribe
}

\author{
Belén Zurbano-Berenguer' (iD) 0000-0002-2743-236X \\ María del Mar García Gordillo' (iD 0000-0002-9367-0366 \\ Alba Zurbano Berenguer ${ }^{2}$ \\ 'Universidad de Sevilla, Sevilla, España, C.P. 41004 \\ ${ }^{2}$ Investigadora independiente
}

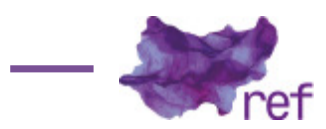

\begin{abstract}
Resumen: Este trabajo estudia las diferencias y similitudes que existen en los diferentes textos legales sobre violencias contra las mujeres en el contexto de América Latina y del Caribe. El objetivo de la investigación es contribuir a los análisis jurídicos de las diferentes legis/aciones para expandir el conocimiento jurídico y poder realizar apuestas legis/ativas de calidad. Los resultados de los análisis, que se basan en las terminologías y conceptualizaciones de las violencias por razón de género, muestran una gran heterogeneidad, reflejo de la falta de consenso social sobre este problema.

Palabras clave: violencia contra las mujeres; violencia de género; derecho; conceptualización; terminología.
\end{abstract}

Violência contra as mulheres nos textos legais da América Latina e do Caribe

Resumo: Este trabalho estuda as diferenças e semelhanças que existem nos diferentes textos legais sobre as violências contra as mulheres no contexto da América Latina e do Caribe. O objetivo da pesquisa é contribuir para a análise jurídica das diferentes legis/ações para ampliar o conhecimento jurídico e fazer apostas legislativas de qualidade. Os resultados das análises, baseados em terminologias e conceituações de violência de gênero, mostram grande heterogeneidade, reflexo da falta de consenso social sobre esta questão.

Palavras-chave: violência contra as mulheres; violência de gênero; direito, conceituação; terminologia.

Violence against Women in Legal Texts in Latin America and The Caribbean

Abstract: This paper studies the differences and similarities that exist in the different legal texts on violence against women in the context of Latin America and the Caribbean. The objective of the research is to contribute to the legal analysis of the different legislations to expand the legal knowledge and to make quality legislative bets. The results of the analysis, which are based on the terminologies and conceptualizations of gender-based violence, show a great heterogeneity that reflects the lack of social consensus on this problem.

Keywords: Violence against women; Gender-based violence; Law; Conceptualisation; Terminology.

\section{Introducción}

Las violencias que se ejercen contra las mujeres (en adelante WCMM) son la primera causa de muerte entre las mujeres de 15 a 44 años por encima de guerras, accidentes de tráfico o cáncer. Las WCMM se destacan, además, por su vertiginosa alta tasa de prevalencia: una de cada tres mujeres en el mundo es golpeada, coaccionada sexualmente o sufre otro tipo de abuso en su vida (UNIFEM, 2003). 
[...] violence against women is pervasive globally. The findings send a powerful message that violence against women is not a small problem that only occurs in some pockets of society, but rather is a global public health problem of epidemic proportions, requiring urgent action. (OMS, 2005; 2013)

Abordar científicamente estas violencias presenta una dificultad de fondo importante: su medición en cifras, es decir, el diseño de los indicadores para dicha medición. La disparidad de cifras barajadas sobre WCMM entre las diferentes instituciones - los gobiernos suelen ofrecer datos menos elevados que las organizaciones feministas (Raquel OSBORNE, 2008, p. 105) - responde a la utilización de diferentes categorías definitorias e indicadores de medición. Numerosas voces se han alzado ya contra la parcialidad y el reduccionismo de las cifras (OSBORNE, 2008). Es en este punto donde la investigación que aquí se presenta pretende explorar un aspecto de gran relevancia: las diferentes conceptualizaciones y terminologías vigentes en el corpus jurídico de violencia de género/contra las mujeres/machista, ${ }^{1}$ entendiendo que éstas son la base jurídicoconceptual que sustenta posteriormente la elaboración de los indicadores de medición y prevalencia de las agresiones violentas (Belén ZURBANO-BERENGUER; Irene LIBERIA, 2014).

Este trabajo ha planteado tres objetivos: 1) conocer las legislaciones existentes en materia de VVCMM en el ámbito latinoamericano; ${ }^{2}$ 2) observar las diferentes conceptualizaciones formuladas y terminologías empleadas en los textos legales nacionales latinoamericanos y 3 ) elaborar una escala de medición de los diferentes grados de complejidad conceptual insertos en los textos normativos que pueda ser útil para aplicar a otros contextos regionales y nacionales. Esta investigación trata, por tanto, de aportar conocimientos nuevos sobre la realidad de las VVCMM, en concreto, sobre su tratamiento jurídico partiendo de la premisa de la función simbólica del Derecho Penal, ${ }^{3}$ por un lado, y de la necesidad y urgencia de nombrar, visibilizar y conceptualizar una realidad que atenta contra los Derechos Humanos y la convivencia democrática (Marcela LAGARDE, 2007; Encarna BODELÓN, 2009).

En este sentido, cabe destacar los encomiables avances realizados desde el feminismo latinoamericano por incorporar al corpus jurídico tipos penales más amplios, abarcadores e inclusivos. ${ }^{4}$ Autoras como Carla Barrio, Estela Santos y Alessandro Gentile (2013) abogan por la complejización y profundización del fenómeno de las WCMM desde un punto de vista conceptual, prestando especial atención a la actividad desarrollada en este sentido por las colegas feministas académicas de América Latina, quienes, desde hace dos décadas, trabajan profusamente sobre esta cuestión: la denominación del problema que supone la(s) violencia(s) ejercida(s) contra las mujeres, formulando nuevas propuestas de análisis como la del feminicidio ${ }^{5}$ y denunciando la brutal violencia que la sociedad tolera y ejerce contra las mujeres.

Es necesario también reconocer al movimiento y la academia feminista latinoamericana el planteamiento que de las WVCMM hacen en términos de negación de derechos ciudadanos, de seguridad pública y de exclusión social, configurándolo en el plano de Teoría Política como un asunto de Justicia y Ciudadanía. Montserrat Sagot subraya en este sentido que "Ia reconceptualización y posicionamiento de la violencia contra las mujeres como un asunto de justicia, ciudadanía y de derechos humanos por parte del movimiento feminista ha implicado importantes avances sociales" (SAGOT, 2008a, p. 222), refiriéndose, entre otros, al avance en términos legislativos.

\section{Fundamentos teóricos de la investigación}

El feminismo es una tradición de pensamiento moral y político y un movimiento social que tuvo su origen en la modernidad y que presentó un contenido igualitario y un carácter emancipatorio por el que ningún individuo debe ser excluido de ningún bien o derecho a causa de su sexo. Además, de acuerdo con Ana de Miguel, se caracteriza por la praxis cognitiva o redefinición de la realidad (subversión de los códigos culturales dominantes). La teoría feminista es una teoría crítica de la sociedad (DE MIGUEL, 2003); una teoría que irracionaliza la visión establecida de la realidad (Celia AMORÓs, 1998), es decir, permite un nuevo acercamiento a la realidad lo cual implica la asunción de un nuevo marco de referencia (Erving GOFFMAN, 1974), desnaturalizando pautas de conductas aprehendidas y valores asumidos. En definitiva, la teoría feminista cuestiona y desvela la realidad patriarcal potenciando la "deslegitimación del entramado conceptual patriarcal o la

\footnotetext{
' Dado el objeto de estudio del presente trabajo, se impone la necesidad de usar con precisión y concienzudamente los términos adecuados que se refieran a la complejidad conceptual que se propone; por ello, se dará prioridad al uso de "violencia machista" o "violencias contra las mujeres" sobre el de "violencia de género".

${ }^{2}$ Siempre que se aluda a la región latinoamericana se estará incluyendo de forma implícita al Caribe.

${ }^{3}$ Cuestión indudablemente controvertida y de gran debate académico que se desarrollará en el apartado siguiente.

${ }^{4}$ Véase el Informe Feminicidio: un fenómeno global. De Madrid a Santiago, que constata el desfase normativo en materia de WVMM entre España (concepción más restringida, circunscrita estrictamente a la violencia entre parejas afectivas y heterosexuales) y Latinoamérica.

${ }^{5}$ El Femicidio, Feminicidio y la Violencia Feminicida se tratarán con mayor detenimiento en el siguiente apartado.
} 
redefinición de la realidad" (DE MIGUEL, 2003, p. 133). En concreto, las aportaciones del feminismo radical de los años setenta son esenciales, ya que posibilitaron visibilizar el problema de las WCMM consiguiendo que la "violencia doméstica" mutara de drama personal a problema social, rompiendo con la dicotomía liberal del espacio (público-privado).

Para el trabajo que aquí nos ocupa, se hace necesario concretar qué rasgos básicos caracterizan las WVMM a las que nos referimos, o, dicho de otra manera, qué hay detrás de la expresión "WCMM", para así poder diferenciarlas de otro tipo de violencias, aspecto éste de vital importancia para refutar los nuevos planteamientos machistas. Para ello, seguimos a Inés Alberdi y Natalia Matas (2002) por la exhaustividad y amplitud de su análisis las cuales proponen 10 elementos definitorios de las WVMM: a) Su origen se encuentra en la asignación de roles diferenciados en función del sexo (sistema sexo-género); b) Es un rasgo social a la vez que un fenómeno individual: es la manifestación de la estructura común patriarcal en el que los hombres, seres individuales, ejercen el dominio; c) Se deriva de las desigualdades de poder entre hombres y mujeres: el patriarcado establece una supremacía masculina; d) Tiene un carácter instrumental: la violencia es el "brazo armado" del patriarcado, es el instrumento para perpetuar el statu quo; e) Es estructural e institucional: esta violencia no se corresponde con determinados estratos sociales ni económicos, sino que es transversal y se legitima institucionalmente en procesos como los de socialización; f) Es ideológica; g) Está por todas partes; h) Afecta a todas las mujeres: como forma de asegurar el dominio masculino todas las mujeres están potencialmente amenazadas por esta violencia, sin embargo, ésta afecta desigualmente a unas y otras dependiendo de diversos factores; i) No es natural, es aprendida: la socialización de género (OSBORNE, 2009, p. 39) opera como mecanismo de asimilación e interiorización de valores, conductas y pautas que legitiman el orden patriarcal. El sexismo es aprendido porque previamente es culturalmente construido; j) Es tolerada socialmente; y k) Pasa desapercibida y es difícil de advertir: la normalización de la violencia dificulta su percepción y denuncia quedando invisibilizada en muchas ocasiones.

Además de esta caracterización, que sirve para definir el objeto de estudio de este trabajo, se considera relevante utilizar como apoyos teóricos los conceptos de "femicidio", "feminicidio" y "violencia feminicida", aportaciones de la academia anglosajona de los años 90 . El término femicidio (en inglés Femicide) fue utilizado por vez primera en 1976 por Diana Russell en el Primer Tribunal Internacional de Crímenes contra las Mujeres. Sin embargo, hubo que esperar hasta 1992 para conocerlo tal y como hoy es concebido: "el asesinato misógino de mujeres cometido por hombres" (Jill RADFORD; RUSSELL, 1992, p. 33). Estas autoras distinguen una amplia variedad de formas de violencia:

[...] violación, tortura, esclavitud sexual (particularmente por prostitución), abuso sexual infantil incestuoso o extra-familiar, golpes físicos y emocionales, acoso sexual (por teléfono, en las calles, en la oficina, y en el aula), mutilación genital [...], operaciones ginecológicas innecesarias [...], heterosexualidad forzada, esterilización forzada, maternidad forzada (por la criminalización de la contracepción y del aborto), psicocirugía, negación de comida para mujeres en algunas culturas, cirugía plástica y otras mutilaciones en nombre del embellecimiento. Siempre que estas formas de terrorismo resultan en muerte, se convierten en feminicidios". (RADFORD; RUSSELL, 1992, p. 57)

Este término ha tenido muy buena acogida en la academia y sociedad latinoamericana, desarrollándose al respecto una importante producción científica (LAGARDE, 2008; Graciela ATENCIO, 2011 ; Patricia LAURENZO, 2012) e incorporándose como terminología y marco conceptual de referencia en numerosas leyes para erradicar o sancionar las WVMM.

Además del anclaje feminista tanto en el abordaje como el objeto de investigación, este trabajo se sustenta en la Teoría Crítica del Derecho. Una Teoría Crítica, recordando a Marx, "es un autoesclarecimiento de las luchas y deseos de una época" y, como sostiene Alda Facio,

para que las críticas que desde los feminismos se le hacen al Derecho puedan considerarse una Teoría Crítica del Derecho, éstas tendrían que tener como objetivo el esclarecimiento del rol que desempeña el Derecho en el mantenimiento del patriarcado. (FACIO, 2000, p. 16-17)

Éste es el punto de partida de esta investigación. La crítica al Derecho androcéntrico surge en el contexto del feminismo radical de los años 70, denunciando la falsa e interesada distinción entre ámbito público y privado; Catherine Mackinnon (1995) representa, junto con otras autoras, la denominada Feminist Jurisprudence, corriente crítica del Derecho que cuestiona la aparente imparcialidad y neutralidad de las estructuras y procedimientos del Derecho; es más, ven en ellas instrumentos al servicio del sistema patriarcal para perpetuar las relaciones de desigualdad. La generalidad y abstracción de las normas y su aplicación formalista han contribuido a afianzar, de forma a priori racional, la posición social de desventaja de las mujeres (Isabel TURÉGANO, 2011).

Aquellos sectores que se muestran favorables a la aprobación de textos legales tendentes a la condena y el castigo (también a la protección, asistencia y reparación de las víctimas) pretenden visibilizar lo que hasta hace relativamente poco tiempo se consideraba una cuestión 
de ámbito privado e íntimo (en el caso de la violencia producida en el ámbito familiar/doméstico) o simplemente desgracias que le suceden a algunas mujeres (agresiones sexuales y acoso sexual laboral), sin conectar todas esas prácticas sociales misóginas toleradas y asumidas tanto por hombres como por mujeres como normales, como naturales. La pretensión de estos sectores del feminismo y operadores jurídicos que abogan por la aprobación de leyes de condena no es otra sino la de, por un lado, repolitizar un espacio configurado desde el pensamiento ilustrado, y, por otro lado, la de conectar las violencias que se ejercen en el ámbito público bajo una estructura de poder desigual basada en el sistema sexo-género, reclamando medidas (en este caso jurídicas y apelando a la función simbólica del Derecho Penal) tendentes al cambio social.

En cambio, otros sectores, reacios a la judicialización (o mejor dicho, suspicaces con una "respuesta penalmente intensa", en palabras de Laurenzo, 2005, p. 23, refiriéndose a la L.0.1/ 2004) como Elena Larrandart (2012), María Luisa Maqueda (2006), Laurenzo (2005), Elena Larrauri (2012), entre otras, esgrimen a su favor, por un lado, no encontrar una correlación entre aumento de la pena y disminución del número de muertes o lo que es lo mismo no creen en el efecto disuasorio del aumento de la pena, y, por otro lado, advierten de los posibles peligros de caer en el error de un ya superado Derecho Penal de Autor (LARRANDART, 2012, p. 183) (que ataca el principio de culpabilidad y el derecho penal del hecho) y de ciertos riesgos como la esencialización de la noción de "Mujer" así como de la anulación de la capacidad de agencia de ésta cayendo en un paternalismo protector estatal (Pastilí TOLEDO, 2009).

Por último, se aborda a continuación una cuestión esencial que ha ido tratándose de forma más o menos explícita a lo largo de las páginas anteriores: la cuestión terminológica y conceptual. El objetivo es apuntar, esclarecer, qué conceptualización de las WVCMM subyace a cada término empleado, qué se está queriendo expresar (u ocultar) cuando se habla de violencia doméstica en lugar de violencia machista, por ejemplo. Se hará un esfuerzo por sintetizar brevemente las terminologías al uso y sus implícitos semánticos e ideológicos.

1. Violencia doméstica. Osborne sostiene que "es el término más pernicioso porque es el que más información oculta" (2009, p. 26). Refiriéndose al ámbito en que sucede "difumina aspectos tan esenciales como son el objeto, el sujeto o el objetivo final de esa violencia" (ÁLVAREZ, 2001, p. 161 apud OSBORNE, 2009, p. 26) escondiendo el hecho de que la mujer agredida lo es por su condición sexual, no por ser madre, esposa, novia o hija (Miguel LORENTE, 1998, p. 85 apud OSBORNE, 1996, p. 26). No diferencia la violencia contra la mujer de la violencia contra niños, ancianos, discapacitados... Y, por supuesto, obvia las violencias que se ejercen en el "espacio público": ámbito laboral, educativo y social.

2. Violencia familiar. Presenta las mismas limitaciones que el término anterior.

3. Terrorismo familiar/ terrorismo sexual/ terrorismo de género/terrorismo patriarcal. Los tres vocablos intentan resemantizar el concepto tradicional de terrorismo tratando de incrementar la fuerza argumentativa de las expresiones que definen esta violencia. En el primero se identifican las mismas limitaciones que para la violencia familiar y la doméstica. El segundo, apunta directamente a las causas de la violencia: el sexo habiendo sido éste generizado pero puede inducir a error con respecto a qué forma toma la violencia (sólo en el plano sexual). El tercero, aunque Lorente (1998, p. 85 apud OSBORNE, 2009, p. 27) lo propuso con la finalidad de distinguir las diferentes víctimas que se cobra, por un lado, terrorismo político (cualquier ciudadano), y, por otro, el terrorismo de género (las mujeres), podría inducir a errores sobre su unidireccionalidad. El cuarto, en cambio, destaca por su alta carga semántica; alude al terrorismo que social y científicamente tiene connotaciones negativas y al patriarcado como causa de esa violencia. Numerosas autoras defienden el uso de este paraguas conceptual del terror para reforzar la idea de violencia estructural, sostenida y brutal que sufren las mujeres (AMORÓs, 2008; FERNÁNDEZ, 2004).

4. Violencia de género. Induce a concebir la violencia como bidireccional (tanto de hombre a mujer como viceversa, de un género a otro y al contrario). No señala el origen o causa de la violencia (el patriarcado) y cae, por tanto, en cierta neutralidad y confusión.

5. Violencia sexista/machista. Determina claramente la causalidad de esa violencia (el sexismo o machismo), quedando claramente establecida sobre quiénes incide (las mujeres por el hecho de serlo). El espacio en que se cometen las violencias no se especifica abarcando, por omisión, tanto al privado como al público.

6. Violencia feminicida. Es una propuesta de Lagarde (2008) que emana del paradigma de análisis del feminicidio. Su utilidad como herramienta analítica es potente, pero de dudosa utilidad práctica en términos sociales, dado que no está extendido su uso. Además, esta violencia solo recoge y alberga para sí los casos más extremos del continuum de la violencia: aquellas mujeres que matan, dejando fuera otras muchas manifestaciones de violencia.

7. Violencia masculina contra la mujer (BONINO, 1999 apud ALBERDI; MATAS, 2002, p. 90). Propuesta por hombres feministas, señala al hombre como el responsable de esa violencia y a la mujer como la receptora.

8. Violencia contra las mujeres. Sitúa en el centro de la violencia a la mujer, pero omite la información sobre perpetradores (¿quién ejerce esa violencia?) y naturaleza de la violencia (¿por 
qué?), además de basar su carga conceptual en las mujeres homogeneizando su identidad como sujeto de opresión (Chandra Talpade MOHANTY, 2008, p. 135).

Tras esta breve revisión terminológica, las autoras del trabajo apostamos por el uso del término "Violencias contra las Mujeres" (NVCMM) (ZURBANO-BERENGUER, 2015) ya que: 1. Es un término claro y pedagógico. Todos los elementos lingüísticos son reconocibles a diferente nivel de formación, lo que permite reconocer claramente el fenómeno; 2. Identifica claramente la unidireccionalidad de la violencia: la violencia la sufren las mujeres por el hecho de serlo; 3. Reconoce las interseccionalidades haciéndose eco de las teorías postfeministas que intentan pluralizar a una mujer que nunca es una sino que son múltiples y diversas; 4 . Apunta la multiplicidad de manifestaciones de las violencias al pluralizarlas, huyendo del paradigma de la agresión física como totalizante y representante de este problema.

Sin embargo, como ha podido observarse, en el campo del Derecho existe gran variedad de términos y conceptualizaciones (unas más amplias, otras más restringidas) para referirse a las WCMM. De ahí que la hipótesis principal de la investigación haya sido que existen diferentes conceptualizaciones jurídicas de las WVMM y, por tanto, diferentes terminologías empleadas. Como subhipótesis se planteó que es posible establecer una gradación entre dichas diferentes conceptualizaciones.

\section{Diseño metodológico}

Para alcanzar los objetivos que esta investigación propone, y con el fin de validar la hipótesis, se traza una estrategia de investigación extensiva: número elevado de casos (29) y bajo de variables (1), dado que lo relevante es la validación, a través de las unidades de observación, de la existencia de diferentes conceptualizaciones, a través de la generación de inferencias descriptivas, para lo cual se realiza un estudio cross-national. A continuación, se exponen de forma detallada las 5 dimensiones y los 15 indicadores que servirán para observar la variabilidad jurídico-conceptual de las WCMM.

1. Tipos de WVMM. Constituyen las manifestaciones a través de las cuales son (en mayor o menor medida) reconocibles las violencias. La consulta de las leyes y otros documentos jurídiconormativos, así como la propia literatura especializada, han ayudado a concretar los tipos de violencias en cinco: física, psíquica, sexual, económica y simbólica. Establecer la línea entre unas y otras es complejo y delicado pues ¿dónde está la frontera entre la violencia física y la sexual en una mutilación genital femenina, por ejemplo? En cualquier caso, esta dimensión parece ser lo suficientemente amplia como para recoger prácticamente la totalidad de las manifestaciones y expresiones de violencia, y lo suficientemente restringida como para ser medible en términos analíticos. En la presente investigación, cuantos más tipos de WCMM (de los cinco mencionados) reconozca la legislación analizada, más amplia podrá entenderse ésta en términos conceptuales y viceversa. Por ello se ha elaborado un sistema de codificación binario $(0,1)$ donde 0 significa que la información no aparece mencionada en la ley, y 1 que sí aparece. ${ }^{6}$ Así pues, en esta dimensión concreta 5 es el mayor valor posible y 0 el menor.

2. Agentes o Ámbitos. Se entiende por "agentes" el/los sujeto/s que ejerce/n las WCMM (antigua o actual pareja, familiares directos o indirectos, superiores o compañeros de trabajo, docentes o compañeros de estudios, agresores o delincuentes desconocidos en la calle, agentes del Estado... Entiéndase por "ámbitos" lugar, espacio o contexto donde son perpetradas esas WCMM. Se desglosa en ámbito privado (que incluye el espacio doméstico) y ámbito público (laboral, educativo, social, estatal). A efectos de codificación se tendrá en cuenta la aparición o ausencia en el texto legal de uno (ámbitos) u otro (agentes) sin tener que ser acumulativos; por ejemplo, es suficiente con que se mencione expareja o ámbito doméstico, no es necesario que figuren los dos. Al igual que en el caso anterior, cuantos más ámbitos o agentes reconozca la legislación se entenderá que al contemplar mayores sujetos susceptibles de infligir violencia y mayores contextos donde perpetrarla, la norma será más inclusiva, es decir, conceptualmente más amplia.

3. Origen. Se refiere a la alusión (o no) del origen, es decir, la naturaleza de las WVCMM (si responde a relaciones desiguales de poder entre hombres y mujeres, al patriarcado...). Si señalan directamente las causas estructurales de las WVMM se entenderá legislación más avanzada, si omite esta información más restringida.

4. Elementos Penales. Esta dimensión se subdivide en dos:

- Naturaleza de la norma. Atiende a si el texto normativo adquiere la forma de ley especial (lo cual suele conllevar medidas aparejadas; normalmente es una política pública más desarrollada e integral que contempla prevención y asistencia) o adquiere la forma de reforma del Código Penal (se limita a sancionar o tipificar). En el primer caso se entenderá como legislación más avanzada y en el segundo más restringida, pues se entiende que se presta una menor atención al problema de las WVMM.

${ }^{6}$ Este sistema de codificación es válido para el resto de las dimensiones. 
- Bien Jurídico que protege la ley. Normalmente responde a la pregunta ¿qué "garantiza" la ley? y suele encontrarse en el "objeto de la ley".

5. Terminología. Se subdivide en dos: título de la norma y delito. Contempla el tipo penal que sanciona la consecuencia más extrema de las WVCMM: la muerte. En concreto se observa si existe tipo penal específico, como por ejemplo la figura de femicidio/feminicidio).

Figura 1. Escala jurídico-conceptual de las violencias contra las mujeres.

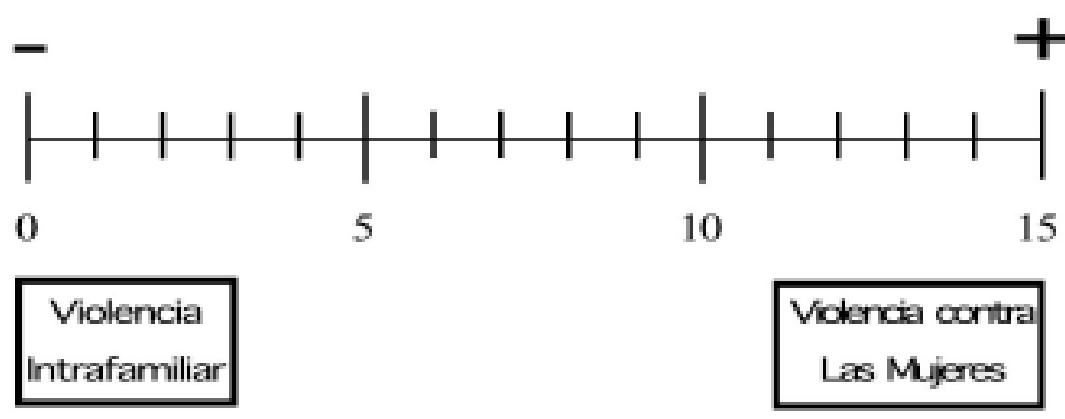

Fuente: Elaboración propia.

\#PraCegoVer En la figura número uno aparece una escala lineal que mide desde el cero hasta el 15 dividiéndose la misma en cuatro tramos: del 0 al 5 , del 5 al 10 y del 10 al 15 . En la parte superior de la escala y sobre el número 0 aparece el símbolo negativo y sobre el número 15 el símbolo positivo. Debajo de la escala y bajo el número 0 aparece recuadrado el concepto "violencia intrafamiliar" y el debajo del número 15 recuadrado el concepto "violencia contra las mujeres".

Se considera, por tanto, que el término "VVCMM" incluye teóricamente más apertura conceptual que otros, entendiéndose por "apertura conceptual" aquella que se refiere a una realidad en la cual es posible identificar más tipos de violencias, más formas de ejercicio de las mismas, más ámbitos de perpetración y mayor diversidad en la categoría "mujer", y que el término "Violencia intrafamiliar" recoge justamente lo opuesto.

\subsection{Diseño muestral y técnicas de recogida y de análisis de información}

Para el propósito que se marca la investigación se ha procedido a un muestreo subjetivo por decisión razonada (Piergiorgio CORBETTA, 2003). El universo está formado por las normas jurídicas vigentes $^{7}$ en materia de WCMM que adopten bien la forma de Ley Especial o bien de reforma del Código Penal, cuyo ámbito de aplicación sea el territorio nacional. La muestra seleccionada ha sido la normativa promulgada en la región de América Latina y el Caribe, con base en los siguientes criterios: uno, variabilidad alta: se han tenido en cuenta los textos normativos circunscritos a esta región porque existe suficiente heterogeneidad entre los casos; y dos, la literatura: la revisión bibliográfica y documental apunta que esta región del mundo está en la punta de lanza de producción jurídica avanzada.

Así pues, se ha observado la legislación de los países de América Latina y el Caribe que cumplían los siguientes criterios: 1. Estar reconocidos como países de América Latina por la CEPAL; 2. Estar constituidos como territorio autónomo y soberano: se estima criterio fundamental que el país, Estado, o nación goce de sus plenas facultades, funciones, poderes legislativos y para ello debe constituirse como territorio soberano y autónomo. ${ }^{9}$ Es necesario mencionar de forma expresa los siguientes casos particulares que quedan excluidos de la muestra por los siguientes motivos: Haití y Cuba carecen de legislación en materia de WCMM, y Dominica y Saint Kitts y las Nieves no disponen de bases de datos actualizadas para los años en que sus legislaciones fueron promulgadas. Se ha solicitado la ley en formato digital a las autoridades pertinentes, pero sin éxito alguno.

- Técnica de recogida de información: la recopilación de los textos legislativos se ha efectuado a través de consultas documentales a fuentes oficiales y públicas durante los meses de febrero a julio de 2014.

\footnotetext{
${ }_{7}^{7}$ No se contemplan proyectos de ley en tramitación, solo legislación aprobada por los cauces legales establecidos por cada país.

${ }^{8}$ Su carácter de organismo adscrito a Naciones Unidas de tipo regional fundamenta que se escogiese ésta para delimitar qué países incluye "América Latina y el Caribe". Disponible en http://bit.ly/1 CpHItr. Última consulta el 10/05/ 2016.

9 Quedan, por tanto, excluidos del análisis los siguientes países: Anguila, Islas Caimán, Islas Turcas y Caicos, Islas Vírgenes Británicas, Islas Vírgenes EEUU y Montserrat, así como, Aruba (territorio de ultramar adscrito al Reino de Países Bajos). Pueden consultarse los países declarados por NNUU como territorios no autónomos en su página web oficial: http://bit.ly/1EgKUcZ. Última consulta el 10/05/2017.
} 
- Técnica de análisis de la información: la técnica empleada para analizar la información ha sido el análisis de contenido realizado a partir de la información sistematizada en una matriz de datos. Para poder ordenar, organizar y procesar la información se ha recurrido a un sistema de codificación binaria. Se asume que el sistema de codificación haya podido limitar, sobre todo, la dimensión terminológica, ya que reduciendo a dos polos (restringido-amplio) la realidad terminológica se pierde riqueza en el análisis, pero como en toda investigación, existen limitaciones materiales, de recursos y de tiempo.

\section{Resultados de la investigación}

Tal y como muestra la Figura 2, los 29 países analizados quedan agrupados a lo largo de la escala en tres grupos principalmente: el primero, que ocupa las posiciones del 0 al 5, lo constituyen 9 países cuyas legislaciones conciben la WVMM de forma restringida; el segundo (posiciones 6-10), está conformado por un grupo de 12 países; y el tercero, lo forma un grupo de 8 países, y la mayor parte de ellos se concentran en las posiciones más altas (14 y 15). Es posible observar que la mayoría de la normativa (12) se encuentra en una posición intermedia en lo que respecta al grado de apertura conceptual en materia de WVMM; así como que los países del primer y tercer grupo tienden, en rasgos generales, a concentrarse en posiciones avanzadas (4 y 5 para el primer grupo y 14 y 15 para el tercero).

A continuación, se procede a señalar sucintamente los resultados obtenidos por países o grupos de países, indicando los valores que toman para cada indicador, comenzando por aquellos que obtienen una puntuación más alta y que, por tanto, según la propuesta que aquí se hace, poseen una conceptualización más amplia de las VVCMM.

Figura 2. Escala jurídico-conceptual de las violencias contra las mujeres en los países latinoamericanos.

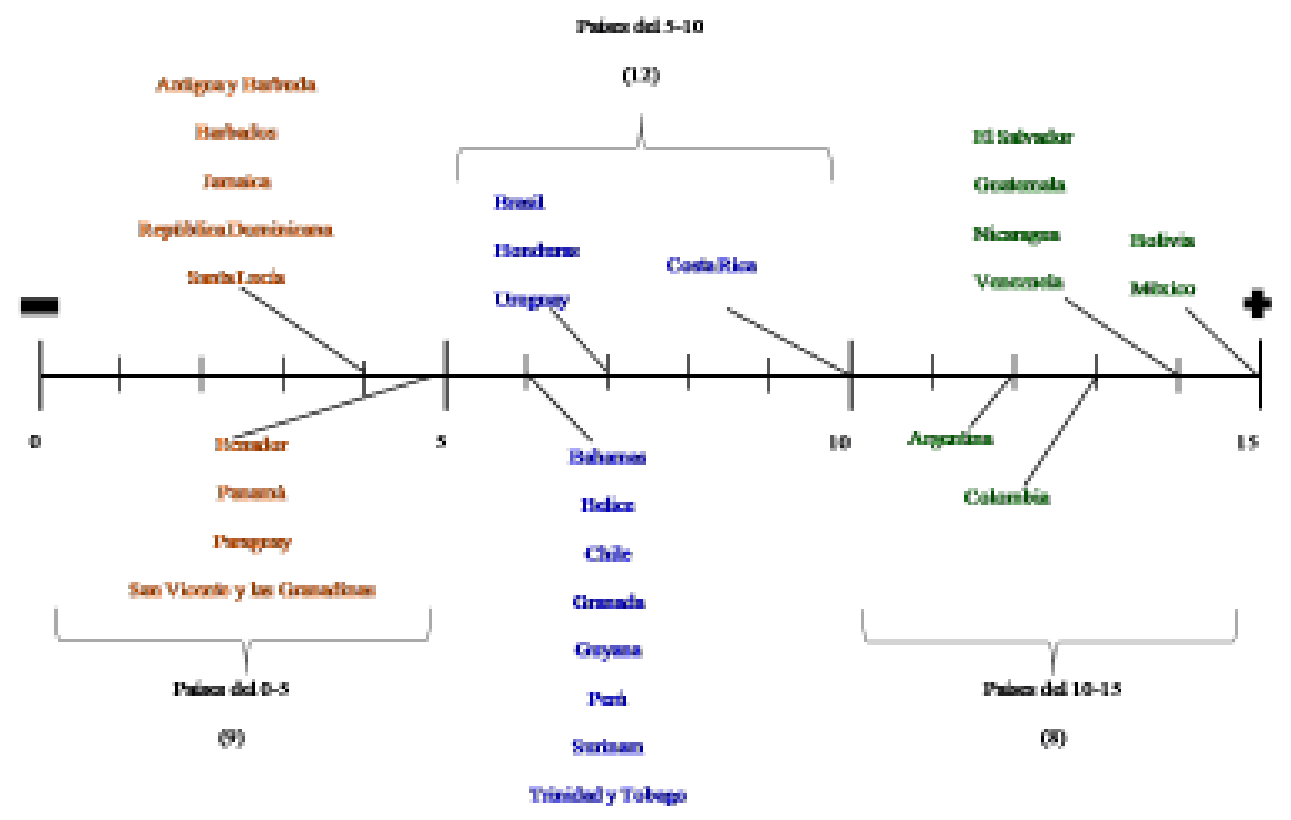

Fuente: Elaboración propia.

\#PraCegoVer En la figura número 2 aparece una escala lineal que mide desde el cero hasta el 15 dividiéndose la misma en cuatro tramos: del 0 al 5, del 5 al 10 y del 10 al 15. En la parte superior de la escala y sobre el número 0 aparece el símbolo negativo y sobre el número 15 el símbolo positivo. En la escala se señalan 15 líneas verticales que se corresponden con cada uno de los 15 puntos de la escala. Sobre la línea que indica el valor 4 en la escala se indican el nombre los países que han obtenido dicha puntuación: Antigua y Barbuda, Barbados, Jamaica, República Dominicana y Santa Lucía. Sobre la línea que indica el valor 5 en la escala se indican el nombre los países que han obtenido dicha puntuación: Ecuador, Panamá, Paraguay, San Vicente y las Granadinas. Sobre la línea que indica el valor 6 en la escala se indican el nombre los países que han obtenido dicha puntuación: Bahamas, Belice, Chile, Granada, Guyana, Perú, Surinam, Trinidad y Tobago. Sobre la línea que indica el valor 7 en la escala se indican el nombre los países que han obtenido dicha puntuación: Brasil, Honduras y Uruguay. Sobre la línea que indica el valor 10 en la escala se indican el nombre del país que ha obtenido dicha puntuación: Costa Rica. Sobre la línea que indica el valor 12 en la escala se indica el nombre del país que han obtenido dicha puntuación: Argentina. Sobre la línea que indica el valor 13 en la escala se indica el nombre del país que han obtenido dicha puntuación: Colombia. Sobre la línea que indica el valor 714 en la escala se indican el nombre los países que han obtenido dicha puntuación: El Salvador, Guatemala, Nicaragua, Venezuela. Sobre la línea que indica el valor 15 en la escala se indican el nombre los países que han obtenido dicha puntuación: Bolivia y Méjico. 
Bolivia y México, con sus respectivas recientes legislaciones (2013 y 2007, respectivamente), encabezan la lista de países que contemplan más formas de manifestación (5 de 5) y ámbitos de perpetración ( 5 de 5) de las violencias y que aluden y destacan el origen patriarcal de dichas violencias ${ }^{10}$. Asimismo, en sus respectivas normativas se hace uso de una terminología más inclusiva (violencia contra las mujeres en ambos casos) y tienen por objeto garantizar a las mujeres una vida digna, el ejercicio pleno de sus derechos para Vivir Bien (BOLIVIA, art. 2) y el acceso a una vida libre de violencia (MÉXICO, art. 1). El análisis efectuado recoge el conjunto de países que obtienen una mejor posición en la escala: El Salvador, Guatemala, Nicaragua y Venezuela, cuyas normativas son todas de reciente promulgación $(2011$, 2008, 2012 y 2007, respectivamente). Como se observa en los resultados, ${ }^{11}$ las reducidas diferencias entre países se encuentran en la omisión de la violencia simbólica en el caso de Guatemala y Nicaragua, de la violencia que se ejerce en el contexto educativo (El Salvador) y del mantenimiento de la misma terminología para el tipo penal que sanciona el homicidio (Venezuela). Asimismo, Colombia y Argentina son países que pueden ser considerdos de legislación avanzada (en el sentido terminológico-conceptual que estainvestigación observa) pues en la inmensa mayoria de los indicadores toma el valor 1, que implica una conceptualización amplia.

Costa Rica, un país que, a pesar de poner de manifiesto el origen patriarcal de las WVMM (tanto en el objeto de su ley como en la tipificación del delito de femicidio -art. 21), destaca por circunscribir las violencias al ámbito doméstico y de la afectividad. ${ }^{12}$ Continuando el descenso por la escala jurídico-conceptual hacia posiciones más restingidas, encontramos, por un lado, países como Brasil, Honduras o Uruguay que apenas hacen alusión a la desigual relación de poder entre sexos como origen de las WVCMM, no se preocupan por el uso de una terminología avanzada y no contemplan la posibilidad de que se ejerzan violencias en ámbitos públicos (laboral, estatal, social y educativo), y, por otro lado, con países que conciben las WVMM como un asunto familiar, por ello el bien jurídico que garantizan en términos generales es "la vida, integridad personal y seguridad de los miembros de la familia" (art. 2 de la ley chilena).

En las últimas posiciones se encuentran aquellos países cuya preocupación jurídicoconceptual en materia de WVCMM se ciñe a la que se produce de forma física, psicológica y sexual y en el ámbito doméstico-familiar, eminentemente: Ecuador, Panamá, Paraguay, S. Vicente y las Granadinas, Antigua y Barbuda, Barbados, Jamaica, República Dominicana y Santa Lucía.

En definitiva, los análisis muestran la heterogeneidad jurídico-conceptual de las normativas analizadas en la región latinoamericana que, como apuntaba la literatura, existe en la actualidad. Cada país, como poder político organizado, autónomo y soberano, muestra mayor sensibilidad y preocupación en unos aspectos y no tanto en otros como ha quedado reflejado en las tablas.

A continuación, se abordará en términos agregados las cinco dimensiones contempladas para la gradación jurídico-conceptual de los textos legales. En primer lugar, como muestra la figura 3 , tan solo unos pocos países (17\%) contemplan en sus legislaciones relativas a WCMM la posibilidad de que se ejerza una violencia simbólica (entre la que se incluye, por ejemplo, la violencia mediática que reproduce y legitima estereotipos sexistas que cosifican a la mujer). Sin embargo, existe amplio consenso normativo-conceptual en concebir y entender la violencia física y la psicológica como manifestaciones de las WVMM: el 100\% de la muestra analizada las contempla.

En segundo lugar, tal y como puede observarse en la figura 4, son relativamente pocos los países que contemplan y recogen en sus legislaciones las violencias producidas en el ámbito laboral, estatal, social y educativo. En cambio, no existe legislación que omita la violencia que se ejerce en el espacio privado.

En tercer lugar, resulta llamativo observar cómo tan solo un 34\% de la legislación promulgada en los países del estudio hace mención expresa al patriarcado (o a las relaciones desiguales de poder entre sexos) como el sistema de dominación que sostiene y auspicia las violencias. Desde el enfoque adoptado en esta investigación, resulta crucial tomar conciencia de por qué se producen las violencias y determinar su origen en pos de un análisis certero de la realidad sobre la que se pretende incidir con el diseño de Políticas Públicas adecuadas.

En relación con los elementos penales, la mayor parte de los países (93\%) cuenta con legislación específica sobre VVCMM (exceptuando Panamá y República Dominicana cuya normativa se ciñe a modificaciones del Código Penal), aunque no todos protegen y garantizan los mismos bienes jurídicos como se ha mostrado en las tablas anteriores relativas a los países: menos de la mitad de las legislaciones (38\%) persiguen proteger o garantizar el derecho a una vida libre

\footnotetext{
${ }^{10}$ La ley boliviana recoge como principio la despatriarcalización de la sociedad e insta a su consecución a través de Políticas Públicas que tengan por objetivo precisamente visibilizar, denunciar y erradicar las relaciones desiguales y de violencia (art. 4).

11 Las indicaciones numéricas están recogidas en los anexos de investigación.

${ }^{12}$ La presente Ley tiene como fin proteger los derechos de las víctimas de violencia y sancionar las formas de violencia física, psicológica, sexual y patrimonial contra las mujeres mayores de edad, como práctica discriminatoria por razón de género, específicamente en una relación de matrimonio, en unión de hecho declarada o no (art. 1).
} 
de violencia, la libertad,la dignidad, o los Derechos Humanos. Ello puede estar conectado a que pocos países aluden al origen patriarcal de las violencias, ya que pocos contemplan la violencia simbólica como manifestación posible.

En última instancia, a través del análisis de la quinta dimensión (terminología normativa) es posible obsevar, por un lado, que tan solo un 34\% de los países estudiados emplea en el título de sus legislaciones un término amplio o avanzado. Resulta importante destacar este aspecto ya que, como se comprobará más adelante, terminología y conceptualización están estrechamente

Figura 3. Tipos de violencias contra las mujeres recogidas en la normativa.

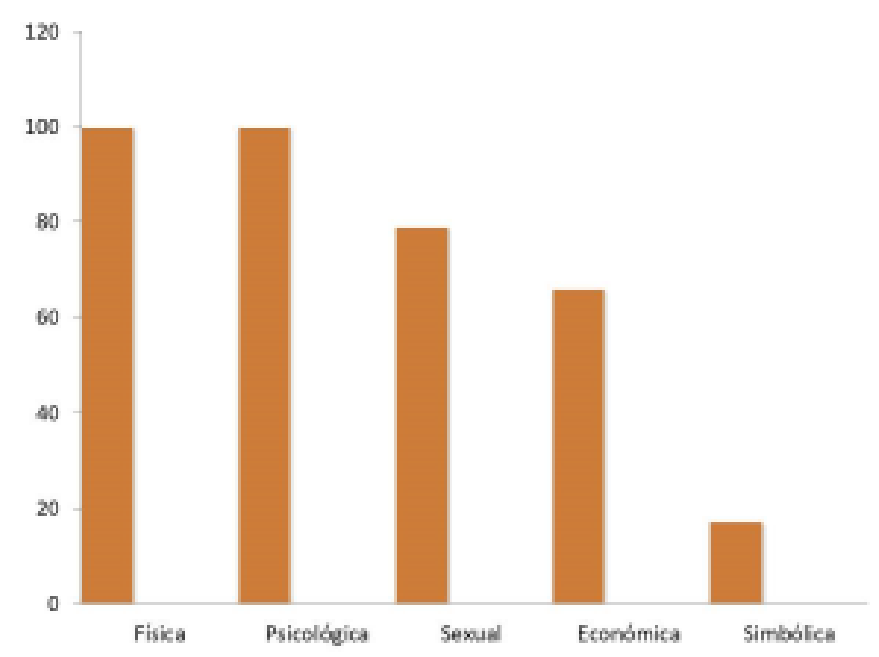

Fuente: Elaboración propia.

\#PraCegoVer En la figura 3 aparece un gráfico de barras ordenado de mayor, la primera barra, a menor, la última. Los colores de la barra van desde el marrón oscuro al naranja degradado de izquierda a derecha. La primera de las columnas indica que el $100 \%$ de las normas contienen alusiones a la violencia física; la segunda columna indica un $100 \%$ de presencia de la violencia psicológica; la tercera columna indica un $79 \%$ de violencia sexual; la cuarta un $66 \%$ de violencia económica y la quinta y última un $17 \%$ de violencia simbólica.

Figura 4. Ámbitos o agentes recogidos en la normativa latinoamericana en materia de violencias contra las mujeres.

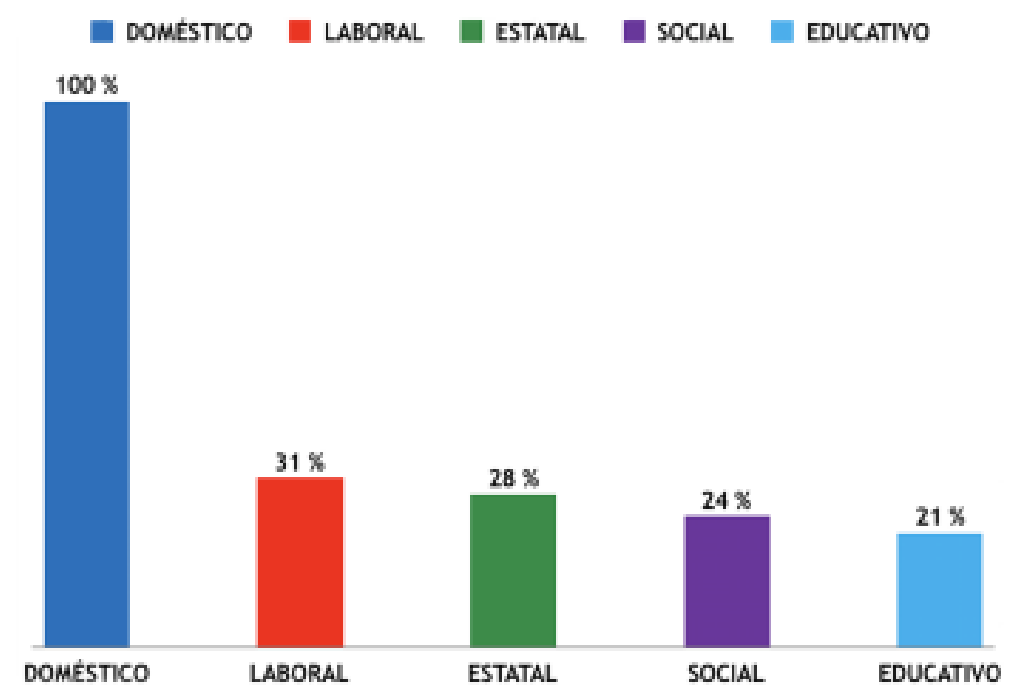

Fuente: Elaboración propia.

\#PraCegoVer En la figura 4 aparece un gráfico de barras ordenado de mayor, la primera barra, a menor, la última. Los colores de la barra son azul, rojo, verde, morado y azul sucesivamente. La primera de las columnas indica que el $100 \%$ de las normas contemplan el ámbito doméstico como espacio de violencia; la segunda columna indica un $31 \%$ de presencia del espacio laboral en estas legislaciones; la tercera columna indica un $28 \%$ de presencia de lo estatal como ámbito de la violencia; la cuarta un $24 \%$ del contexto "social" y la quinta y última un $21 \%$ del espacio educativo. 
relacionados. Y, por otro lado, se observa que pocas legislaciones modifican terminológicamente sus tipificaciones (para el delito de homicidio).

A grandes rasgos, este trabajo se planteaba en su comienzo si la normativa latinoamericana pudiera albergar una pluralidad jurídico-conceptual en cuanto a las WCMM y si existiera una terminología diferenciada en dicha materia. Los resultados presentados hasta el momento parecen corroborar esa hipótesis, y la agregación de datos permite presentar los siguientes hallazgos al respecto:

1. Efectivamente, la terminología al uso varía respecto de una legislación a otra siendo las más comunes "violencia doméstica" y "violencia contra las mujeres" y la menos frecuente "violencia familiar".

2. Existe una diferente conceptualización de las WCMM en las diferentes normativas que oscila entre los 0 y 15 puntos contemplados en la escala de medición, con una amplia gama de posiciones intermedias.

3. Es posible observar una correlación positiva entre terminología avanzada y conceptualización amplia que se corresponde asimismo con las legislaciones de mayor actualidad, las promulgadas más recientemente.

Puede observarse que la terminología empleada varía de una legislación a otra, que no existe una homogeneidad terminológica (ni conceptual como se mostrará a continuación) en los textos legislativos, siendo la fórmula más extendida "violencia doméstica" (58\%), aunque tal y como se refleja en los análisis efectuados no se corresponde con legislación de reciente promulgación, la cual sí emplea términos más avanzados.

En segundo lugar, la conceptualización inserta en los textos legales, es decir, qué entienden los legisladores (quedando plasmado en la ley) por la fórmula "VVCMM", varía de una normativa a otra, según se observa en la amplia variedad de puntuación obtenida (desde los 4 puntos deAntigua y Barbuda, Barbados, Jamaica, República Dominicana y Santa Lucía, hasta los 15 de Bolivia y México) en la escala de medición. Que los países recojan en sus respectivas normativas conceptos legales más avanzados y amplios implica que contemplan más tipos de violencia (no solo la física, psicológica y sexual: triada clásica), más ámbitos de perpetración (no solo el doméstico sino también la escuela, el trabajo o los hospitales y cárceles, por ejemplo), más sujetos susceptibles de ejercer violencia (va más allá de la violencia en la pareja) y mayor interés por proteger la dignidad de la mujer y sus derechos humanos y no tanto la unidad familiar.

El salto de una concepción a otra (aunque no quede perfectamente delimitada la frontera entre una y otra habiendo situaciones intermedias) es cualitativamente importante. Es posible apreciar las diferencias de forma visual en la anterior Figura 2, en la cual los países se sitúan a lo largo de la escala en función de la puntuación obtenida (1-15), de este modo observándose los diferentes grados y niveles de conceptualizaciones jurídicas que de las WVMM recogen en sus respectivas legislaciones.

En tercer lugar, tal y como se ha avanzado anteriormente, se observa una correlación directa entre conceptualización amplia y terminología avanzada que se corresponde o coincide con las normativas de más reciente promulgación. La legislación con terminología avanzada y conceptualización amplia en esta materia de las WVCMM son las últimas promulgadas -desde 2007 (México, Venezuela, Costa Rica) hasta 2013 (Bolivia)-, por tanto, es posible identificar una tendencia creciente hacia el empleo de fórmulas terminológicas más inclusivas, aperturistas y avanzadas, como pone de manifiesto el uso exponencial de la voz "violencia contra las mujeres" (29\%). Ello parece apuntar un importante cambio en la definición del problema público, dado que el uso de la expresión "VVCMM" significa entender y comprender este fenómeno de forma compleja, estructural y multidimensional. El siguiente gráfico muestra cómo una conceptualización más amplia de las WVMM se corresponde con una terminología más avanzada.

\section{Conclusiones}

A la luz de los resultados expuestos podría concluirse que el presente trabajo ha permitido ofrecer un conocimiento sobre la legislación vigente y las diferentes conceptualizaciones y terminologías al uso en materia de WCMM, validando la hipótesis inicial que la literatura señalaba: existen nuevas conceptualizaciones de las WCMM y es posible establecer una gradación entre los diferentes conceptos legales que el corpus jurídico latinoamericano contempla. Con el presente trabajo se ha realizado una aproximación estática al resultado final de los cambios acaecidos en lo jurídico: la ley, pero, en términos analíticos, sería tremendamente interesante tratar de explicar por qué, a qué se debe, que dichas leyes se estén aprobando en las respectivas cámaras legislativas; es decir, determinar o tratar de identificar los actores, procesos y mecanismos que permiten y facilitan la promulgación de estas leyes recientes de marcado carácter progresista.

La literatura existente sobre los factores o variables que pueden influir en la elaboración de una política pública sobre WVCMM señala principalmente dos posibles elementos causales: la representación (descriptiva o sustantiva) de las mujeres en los parlamentos (poder legislativo) y la 
autonomía y fortaleza del movimiento(s) feminista(s). En el primer caso, la literatura es amplia, extensa y altamente documentada, en cambio, la segunda perspectiva de análisis constituye un incipiente campo de trabajo (Mala HTUN; Laurel S. WELDON, 2012).

Asimismo, también adquiere importancia notable un análisis más exhaustivo (no solo en términos conceptuales y terminológicos, como es el caso de esta investigación) de las legislaciones que contemple la observación de las diferentes medidas planteadas para erradicar las violencias, así como su posible impacto. En cualquier caso, esta investigación supone un punto de partida y ofrece una información (si bien de tipo regional: América Latina y el Caribe) sobre el estado de la cuestión conceptual - terminológica en el ámbito jurídico que posibilita continuar con el estudio de las WCMM desde diferentes perspectivas.

\section{Referencias}

ALBERDI, Inés; MATAS, Natalia. La violencia doméstica. Informe sobre los malos tratos en España. Barcelona, Spain: Fundación La Caixa, 2002.

AMORÓS, Celia. Tiempo de feminismo. Madrid, España: Cátedra, 1998.

AMORÓS, Celia. "Conceptualizar es politizar". In: LAURENZO, Patricia; MAQUEDA, María Luisa; RUBIO, Ana María (Coord.). Género, violencia y derecho. Valencia, España: Tirant lo Blanch, 2008. p. 15-25.

ATENCIO, Graciela. Femicidio-feminicidio. "Un paradigma para el análisis de la violencia de género". Feminicidio.net. 201 1. Disponible en http://goo.gl/t90oK. Acceso 18/10/2016.

BARRIO, Carla; SANTOS, Estela; GENTILE, Alessandro. El discurso sobre el feminicidio en la sociedad civil española. In: CONGRESO DE LA FEDERACIÓN ESPAÑOLA DE SOCIOLOGÍA. Madrid, España, 2013.

BODELÓN, Encarna. "Las mujeres y las nuevas legislaciones sobre sus derechos: el caso del derecho a la seguridad". Revista Catalana de Seguridad Pública, Barcelona, n. 20, p. 73-84, 2009. Disponible en http://bit.ly/1CbWOlz. Acceso 18/10/2016.

CORBETTA, Piergiorgio. Metodología y Técnicas de Investigación Social. Madrid: McGraw Hill, 2003.

DE MIGUEL, Ana. "El movimiento feminista y la construcción de marcos de interpretación. El caso de la violencia contra las mujeres". Revista Internacional de Sociología (RIS), Madrid, v. 3, n. 35, p. 127-150, 2003.

FACIO, Alda. "Hacia otra teoría crítica del Derecho". In: HERRERA, Gioconda. (Coord.). Las fisuras del patriarcado: reflexiones sobre feminismo y derecho. Ecuador: FLACSO, 2000. p. 15-44.

FERNÁNDEZ, Concepción. "Violencia contra las mujeres: una visión estructural". Intervención Psicosocial, Madrid, v. 13, n. 2, p. 155-164, 2004.

GOFFMAN, Erving. Frame Analysis. Boston, USA: Northeastem University Press, 1974.

HTUN, Mala; WELDON, S. Laurel. "The Civic Origins of Progressive Policy Change: Combating Violence against Women in Global Perspective, 1975-2005". American Political Science Review, v. 106, n. 3, p. 548-569, 2012.

LAGARDE, Marcela. "Por los derechos humanos de las mujeres: la Ley General de Acceso de las Mujeres a una Vida Libre de Violencia". Revista Mexicana de Ciencias Políticas y Sociales, Ciudad de México, v. 49, n. 200, p. 143-165, 2007.

LAGARDE, Marcela. "Antropología, feminismo y política: violencia feminicida y derechos humanos de las mujeres". In: BULLEN, Margaret Louise; DÍEZ, María Carmen (Coords.). Retos Teóricos y Nuevas Prácticas. España: Ankulegi, 2008. p. 209-240.

LARRAURI, Elena. "Violencia de género en España. Tres años después de la lo 1/2004 de 28 de diciembre de Medidas de protección integral contra la violencia de género". In: BIRGIN, Haydée; GHERARDI, Natalia (Coords.). Género, Derecho y Justicia: Vol. 7. Reflexiones jurídicas desde la perspectiva de género, 2012. p. 103-150.

LAURENZO, Patricia. "La violencia de género en la ley integral: valoración político-criminal". Revista Electrónica de Ciencia Penal y Criminología, Granada, n. 8, p. 1-23, 2005.

LAURENZO, Patricia. "Apuntes sobre el Feminicidio". Revista de Derecho Penal y Criminología, Madrid, v. 3, n. 8, p. 119-143, 2012. 
MACKINNON, Catherine. Hacia una teoría feminista del Estado. España: Cátedra, 1995.

MAQUEDA, María Luisa. "La violencia de género: entre el concepto jurídico y la realidad social". Revista Electrónica de Ciencia Penal y Criminología, Granada, n. 8, p. 1-13, 2006. ISSN 16950194.

MOHANTY, Chandra. "Bajo los ojos de Occidente. Academia Feminista y discurso colonial". Traducción de María Vinós. In: SUÁREZ, Liliana; HERNÁNDEZ, Aída (Eds.). Descolonizando el feminismo. Teorías y prácticas desde los márgenes. Madrid: Cátedra, 2008. p. 112-161.

ORGANIZACIÓN MUNDIAL DE LA SALUD. Resumen de informe. Estudio multipaís de la OMS sobre salud de la mujer y violencia doméstica. Primeros resultados sobre la prevalencia, eventos relativos a la salud y respuestas de las mujeres a dicha violencia. Suiza: Departamento Género, Mujer y Salud, 2008.

ORGANIZACIÓN MUNDIAL DE LA SALUD (OMS) Estimaciones mundiales y regionales de la violencia contra la mujer. Italia: Organización Mundial de la Salud, 2013.

OSBORNE, Raquel. "De la "violencia" (de género) a las 'cifras de la violencia': una cuestión política". Empiria: Revista de metodología de ciencias sociales, Madrid, n. 15, p. 99-124, 2008.

OSBORNE, Raquel. Apuntes sobre Violencia de Género. España: Bellaterra, 2009.

RADFORD, Jill; RUSSELL, Diana. Femicide: The Politics of Woman Killing. Nueva York, USA: Twayne Pub, 1992.

SAGOT, Montserrat. "Estrategias para enfrentar la violencia contra las mujeres: reflexiones feministas desde América Latina". Athenea Digital, Barcelona, n. 14, p. 215-228, 2008 a.

TOLEDO, Pastilí. Feminicidio. México: Oficina en México del Alto Comisionado de las Naciones Unidas para los Derechos Humanos (OACNUDH), 2009. Disponible en https://goo.gl/AFyPKP. Acceso el 18/10/2016.

TURÉGANO, Isabel. "Derecho y violencia contra las mujeres: la perspectiva feminista". In: ZURRILLA, María de los Ángeles; MARTíNEZ, Pilar (Coords.). Violencia contra las mujeres. Un enfoque jurídico. España: Septem Ediciones, 2011. p.13-46.

UNITED NATIONS DEVELOPMENT FUND FOR WOMEN (UNIFEM). Not a minute more. Ending violence against women. Nueva York, EEUU: United Nations Development Fund for Women, 2003.

ZURBANO, Belén; LIBERIA, Irene. "Revisión teórico-conceptual de la violencia de género y de su representación en el discurso mediático. Una propuesta de resignificación". ZER, Bilbao, Universidad del País Vasco, n. 36, p. 121-143, 2014.

Belén Zurbano-Berenguer (bzurbano@us.es) es Investigadora y profesora en estudios de comunicación, género y violencias. Ha impartido docencia en la Universidad de Sevilla, en la Universidad del País Vasco, en la Universidad Pablo de Olavide, en la Université Moulay Ismail (Mèknes, Marruecos) y en la Universidad de Málaga. Su trabajo académico está especializado en el estudio de las violencias contra las mujeres desde un enfoque sociológico, jurídico y comunicativo.

María del Mar García Gordillo (marggordillo@us.es) es Profesora titular del Departamento de Periodismo Il de la Facultad de Comunicación de Sevilla. Ha impartido docencia en el Grado en Periodismo, el Máster de Comunicación Política e Institucional, Máster en Comunicación y Cultura, Máster en Guión y en el Máster en Estudios Europeos. Es Directora General de Comunicación de la Universidad de Sevilla.

Alba Zurbano Berenguer (alba.zurbano@gmail.com) es Licenciada en Ciencias Políticas y Administración y una Maestría en Estudios Sociopolíticos, ambos de la Universidad Pablo de Olavide. Su formación universitaria se ha centrado en los estudios feministas y específicamente en el diseño de indicadores con perspectiva de género. Sus aspectos profesionales destacados son su participación en el diseño y preparación de seminarios y talleres europeos dedicados al empoderamiento. 


\section{COMO CITAR ESTE ARTíCULO, DE ACUERDO CON LAS NORMAS DE LA REVISTA:}

ZURBANO-BERENGUER, Belén; GORDILLO, María del Mar García; BERENGUER, Alba Zurbano. "Las violencias contra las mujeres en los textos jurídicos de América Latina y el Caribe". Revista Estudos Feministas, Florianópolis, v. 27, n. 3, e54442, 2019.

\section{CONTRIBUCIÓN DE AUTORÍA}

Todas las autoras han procedido al diseño conceptual y metodológico del artículo. Alba Zurbano Berenguer se ha encargado específicamente de la recolección y la sistematización de los datos. María del Mar García Gordillo ha aportado mejoras a su explotación y a la supervisión de la redacción final. Belén Zurbano Berenguer ha coordinado los enfoques teóricos y la interpretación de los hallazgos. En suma, ha sido un trabajo desarrollado por todas las autoras de forma colegiada.

\section{FINANCIACIÓN}

No aplicable.

\section{CONSENTIMIENTO DE USO DE IMAGEM}

No aplicable

\section{APROBACIÓN DE COMITÉ DE ÉTICA EN INVESTIGACIÓN}

No aplicable

\section{CONFLICTO DE INTERESES}

No aplicable.

\section{LICENCIA DE USO}

Este artículo está licenciado bajo la Licencia Creative Commons CC-BY Internacional. Con esta licencia se puede compartir, adaptar, crear material para cualquier objetivo, siempre que se le atribuya la autoría.

\section{HISTORIAL}

Recebido em 07/12/2017

Reapresentado em 20/09/2018

Aprovado em 27/04/2019

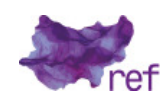

